\title{
Assessment of Heart Rate Variability in Patients with Fibroid Uterus
}

\author{
Dhanalakshmi Y ${ }^{1}$, Pal. G. K², Sirisha.A ${ }^{3}$, Jothi Marie Feula.A ${ }^{4}$, Saranya K ${ }^{5}$, Bhavya R.L ${ }^{4}$, Prethissha $\mathbf{S}^{6}$ \\ ${ }^{1}$ Associate Professor, ${ }^{2}$ Senior Professor, ${ }^{3} \mathrm{PhD}$ Scholar, ${ }^{4}$ Senior Resident, ${ }^{5}$ Assistant Professor, Dept of \\ Physiology, ${ }^{6}$ MSc. Medical Physiology, Post Graduate, Dept of Physiology, Jipmer, Puducherry
}

\begin{abstract}
Background \& Aims: Uterine leiomyoma's represent $29.4 \%$ and $41.4 \%$ of the hysterectomies in women aged

18-44 and 45-64, respectively. They are characterized by an increase in smooth muscle cell proliferation and excessive deposition of extracellular matrix proteins, collagens type I and III. The reactive oxygen species (ROS) has been shown to be involved in the signaling pathways of several growth factors that stimulate proliferation of a variety of cell types. The underlying inflammation leads to the genesis of benign tumors and cardiac autonomic dysfunction. The cumulative effect of oxidative stress, inflammation and ageing make these patients more vulnerable for cardiovascular autonomic imbalance. Therefore, the study was conducted to assess the heart rate variability as a non-invasive tool for assessing cardiac autonomic function in these patients.
\end{abstract}

Method: Thirty four female patients in the age group of $25-50$ yrs with newly diagnosed uterine fibroid were recruited from the department of Obstetrics and Gynaecology of JIPMER. Thirty four female healthy volunteers aged between 25-50 years were recruited as controls. The parameters measured were Basal Heart rate (BHR), Systolic blood pressure (SBP), Diastolic blood pressure (DBP) and Heart rate variability parameters (time and frequency domain indices).

Results: SBP and diastolic BP were elevated in fibroid patients compared to controls. SBP was statistically significant ( $\mathrm{P}$ value $<0.001$ ). Time domain indices were significantly reduced (SDNN: $\mathrm{P}<0.001, \mathrm{RMSSD}$ : $\mathrm{P}$ $<0.001$, PNN50: 0.001) in fibroid patients. Among frequency indices, Total power was significantly reduced $(\mathrm{P}<0.001)$, LFnu was significantly high $(\mathrm{P}<0.001)$, HFnu was significantly decreased $(\mathrm{P}<0.001)$ and LF-HF ratio was significantly increased $(\mathrm{P}<0.001)$ in fibroid patients.

Conclusion: We conclude that fibroid patients have altered autonomic tone in the form of increased sympathetic tone and decreased parasympathetic tone.

Keywords: Fibroid, heart rate variability, autonomic imbalance.

\section{Background}

Uterine leiomyomas represent $29.4 \%$ and $41.4 \%$ of the hysterectomies in women aged 18-44 and 45-

\section{Corresponding author:}

Dr. Y.Dhanalakshmi

Associate Professor, Dept of Physiology, Jipmer, Puducherry.
64, respectively ${ }^{(1)}$. Uterine leiomyomas, or fibroids, are characterized by an increase in smooth muscle cell proliferation and excessive deposition of extracellular matrix proteins, primarily collagens type I and III (2, ${ }^{3,4)}$. Oxidative stress is considered to be involved in pathogenesis of many disorders of the female genital tract ${ }^{(5)}$. The reactive oxygen species (ROS) producing NADPH oxidase complex has been shown to be involved in the signaling pathways of several growth 
factors, cytokines, and vasoactive agents that stimulate proliferation of a variety of cell types ${ }^{(6)}$. Oxidative stress marker level in the serum and tissue samples of fibroid uterus have been documented in earlier studies (7). This was further evidenced from reports on decrease in size of the leiyomyoma following supplementation of antioxidants (Epigallocatechin gallate), which resulted in decrease in the levels of malondialdehyde MDA and TNF- $\alpha{ }^{(8)}$. The cumulative effect of oxidative stress, inflammation and ageing make these patients more vulnerable for cardiovascular autonomic imbalance. However, till date no study has been conducted to assess the autonomic dysfunction in uterine fibroids. Therefore, in this study we propose to assess the cardiovascular autonomic functions in fibroid uterus patients and age and BMI matched subjects and their correlation with the level of oxidative stress and inflammatory markers. Therefore, the study was conducted to assess the heart rate variability as a non-invasive tool for assessing the cardiac autonomic function in these patients.

\section{Objectives of the Study:}

- To assess the Heart rate Variability in Fibroid uterus patients

- To compare the heart rate variability parameters between fibroid uterus patients and healthy controls.

\section{Materials \& Method}

\section{- Inclusion criteria}

After obtaining permission from the institute ethics committee, thirty four female patients in the age group of $25-50$ yrs with newly diagnosed uterine fibroid were recruited from the department of Obstetrics and Gynaecology of JIPMER.

Thirty four healthy female volunteers aged between 25 and 50 years were recruited as controls.

\section{- Exclusion criteria:}

- Patient with other gynaecological problems

- Patients with Hypothyroidism, Hypertension, Diabetes mellitus, Menstrual irregularities, hormonal therapy

\section{Statistical analysis}

The sample size was calculated using Open Epi version 2 Software. Assuming 30\% prevalence of fibroid uterus in women beyond 30 years of age, with 95\% CI and 10\% allowable error (Absolute precision) the sample size calculated was 34 in each group

\section{Recording of HRV}

- Subjects were asked to lie down in supine position. After 15 minutes of supine rest baseline heart rate and blood pressure were recorded using oscillator (Omron MX3). Lead IIECG electrodes were connected from the subject to polygraph .After 10minutes. From Lead II ECG, RR intervals were extracted and analysed using Kubios Software. Time domain parameters were derived from normal R-R interval. Frequency domain HRV indices were obtained by Power Spectral Density (PSD) analysis using Fast Fourier transformation.

\section{Results}

SBP was significantly high (table-1), statistically significant and diastolic high though statistically not significant in fibroid patients compared to controls. Time domain indices (Table -3) were significantly reduced (SDNN: $\mathrm{P}<0.001$, RMSSD: $\mathrm{P}<0.001$, PNN50: 0.001) in fibroid patients when compared to controls. Among frequency indices (Table -2), Total power was significantly reduced $(\mathrm{P}<0.001)$, LFnu was significantly high $(\mathrm{P}<0.001)$, HFnu was significantly decreased $(\mathrm{P}<0.001)$ and LF-HF ratio was significantly increased $(\mathrm{P}<0.001)$ in fibroid patients. 


\section{BASAL PARAMETERS}

(Table-1) Comparison of baseline parameters between study and the control group.

\begin{tabular}{|c|c|c|c|}
\hline Parameter & $\begin{array}{l}\text { Fibroid Uterus } \\
\qquad(\mathbf{N}=\mathbf{3 4})\end{array}$ & $\begin{array}{c}\text { Controls } \\
(\mathbf{N}=\mathbf{3 4})\end{array}$ & p Value \\
\hline Age & $38.411 \pm 6.035$ & $28.688 \pm 7.350$ & 0.0001 \\
\hline BMI & $23.278 \pm 4.064$ & $24.326 \pm 4.457$ & 0.314 \\
\hline SBP & $112 \pm 12.027$ & $103.469 \pm 11.648$ & $0.004^{*}$ \\
\hline DBP & $70.176 \pm 8.547$ & $66.500 \pm 8.710$ & 0.08 \\
\hline HR & $82.088 \pm 12.263$ & $73.313 \pm 10.870$ & 0.0027 \\
\hline PP & $41.823 \pm 7.229$ & $36.969 \pm 9.114$ & 0.017 \\
\hline MAP & $84.117 \pm 9.236$ & $78.823 \pm 8.795$ & 0.0182 \\
\hline RPP & $92.327 \pm 19.087$ & $76.411 \pm 17.020$ & 0.0006 \\
\hline
\end{tabular}

Analysed by unpaired ' $\mathrm{t}$ ' test. *P values less than 0.05 were considered statistically significant

\section{HRV- FREQUENCY DOMAIN PARAMETERS}

(Table-2) Comparison of HRV frequency domain parameters between study and control group

\begin{tabular}{|c|c|c|c|}
\hline arameter & $\begin{array}{l}\text { Fibroid Uterus } \\
\qquad(\mathbf{N}=\mathbf{3 4})\end{array}$ & $\begin{array}{c}\text { Controls } \\
(\mathbf{N}=\mathbf{3 4})\end{array}$ & p Value \\
\hline VLF & $117.281 \pm 82.553$ & $70.40 \pm 80.259$ & 0.0001 \\
\hline LF & $236.188 \pm 119.071$ & $94.697 \pm 117.475$ & 0.0001 \\
\hline $\mathrm{HF}$ & $108.276 \pm 174.903$ & $325.875 \pm 174.242$ & 0.0001 \\
\hline $\mathrm{TP}$ & $273.382 \pm 335.565$ & $679.344 \pm 321.190$ & 0.0001 \\
\hline LF nu & $54.597 \pm 17.837$ & $44.900 \pm 10.607$ & 0.008 \\
\hline HF nu & $45.402 \pm 17.837$ & $55.100 \pm 10.607$ & 0.008 \\
\hline LF:HF & $1.639 \pm 1.386$ & $0.758 \pm 0.343$ & 0.005 \\
\hline
\end{tabular}




\section{HRV- TIME DOMAIN PARAMETERS}

(Table-3) Comparison of HRV time domain parameters between study and control group

\begin{tabular}{|c|c|c|c|}
\hline Parameter & $\begin{array}{l}\text { Fibroid Uterus } \\
\quad(\mathbf{N}=\mathbf{3 4})\end{array}$ & $\begin{array}{c}\text { Controls } \\
(\mathbf{N}=\mathbf{3 4})\end{array}$ & p Value \\
\hline Mean RR & $0.730 \pm 0.158$ & $0.818 \pm 0.122$ & 0.0154 \\
\hline SDNN (ms) & $30.441 \pm 18.986$ & $46.188 \pm 23.870$ & 0.001 \\
\hline RMSSD & 24. $279 \pm 16.593$ & $49.181 \pm 20.606$ & 0.001 \\
\hline NN50 & $20.617 \pm 40.482$ & $79.313 \pm 40.350$ & 0.0001 \\
\hline p NN50 & $5.147 \pm 10.385$ & $39.022 \pm 17.031$ & 0.0001 \\
\hline
\end{tabular}

Analysed by unpaired ' $\mathrm{t}$ ' test. $* \mathrm{P}$ values less than 0.05 were considered statistically significant

\section{Discussion}

The regulation of heart rate relies on the balance between sympathetic and parasympathetic branches of the autonomic nervous system ${ }^{(10)}$. Though there are numerous methods to assess the autonomic modulation of the cardiovascular system, HRV has been one of the most reliable non-invasive method to evaluate heart rate regulation.

Oxidative stress has been implicated in cardiovascular autonomic imbalance. A relation also exists between an impaired immune system, especially the process of inflammation and the pathogenesis of these tumors ${ }^{(7)}$. Also in normal women, alterations of mitochondrial bioenergetics in the heart, consequence from normal aging process, result in decreased fatty acid oxidation and accumulation of fatty acid intermediates in the cardiacmyocyte cytosol, resulting in lipotoxicity and increases the cardiovascular risk ${ }^{(6)}$.

The pathophysiology of uterine leiomyomas is similar to that of other fibrotic conditions such as atherosclerosis, vascular restenosis, and liver, pancreatic, and renal interstitial fibrosis, in which an injury triggers the quiescent cells to dedifferentiate into a myofibroblast-cell like, more proliferative phenotype ${ }^{(7,8)}$. Inadequate sympathetic preponderance and stimulation in individuals with uterine fibroids independent of sex steroids was observed in study conducted by Yun AJ etal $(2005)^{(9)}$. Our study corroborates with the findings of Yun AJ et al. Increased exposure of the uterine environment to seminal fluid that contains catecholamines, aldosterone, prostaglandins and earlier age of pregnancy enables helper $\mathrm{Th}(2)$ cell activation and decreased fibroid growth. Also exposure of the uterine environment to intra uterine devices enabled $\mathrm{Th}\left({ }_{1}\right)$ helper mediated immune response to foreign body may also be attributed to the genesis of fibroid in the study subjects. Also decreased intercourse also could be one of the attributes. Sympatheic function variables seem to be increased amongst the subjects in comparison to the parasympathetic function which may be due to the sex steroid influence. Lesser exposure of the uterine environment to the seminal fluid could be another reason for the increased sympathetic response. Also increased non specific inflammatory markers could influence the sympathetic nervous system. Though statistically not significant the diastolic blood pressure was observed to be high in the study group as compared to the controls due to sympathetic preponderance.

\section{Conclusion}

We conclude that fibroid patients have altered autonomic tone in the form of increased sympathetic tone and decreased parasympathetic tone. 
Conflict of Interest: We authors declare that we do not have any conflict of interest.

Source of Funding: Nil.

Ethical Clearance: We have obtained ethical clearance from the institute ethics committee for conducting this study.

\section{References}

1. Merrill RM. Hysterectomy Surveillance in the United States, 1997 Through. 2005. Med Sci Monit 2008; 14: CR24-CR31.CR31.

2. Paulo J. Oliveira, Rui A. Carvalho, PieroPortincasa, LeonildeBonfrate and VilmSardao. Fatty Acid Oxidation and Cardiovascular Risk during Menopause: A Mitochondrial Connection?.J Lipids. 2012; 2012: 365798.

3. Stewart EA, Friedman AJ, Peck K, Nowak RA. Relative overexpression of collagen type I and collagen type III messenger ribonucleic acids by uterine leiomyomas during the proliferative phase of the menstrual cycle. J ClinEndocrinolMetab 1994; 79: 900- 906.906.

4. Walker CL, Stewart EA. Uterine fibroids: the elephant in the room. Science 2005; 308: 1589 1592.1592 .

5. Pejic S, Kasapovic J, Todorovic A, Stojiljkovic V, Pajovic SB. Lipid peroxidation and antioxidant status in blood of patients with uterine myoma, endometrial polypus, hyperplastic and malignant endometrium.Biol Res. 2006;39(4):619-29. Epub 2007 Jul 20

6. Fernando S. Mesquita, Summer N. Dyer, Daniel A. Heinrich, Serdar E. Bulun, Erica E. Marsh, and Romana A. Nowak. Reactive Oxygen Species Mediate Mitogenic Growth Factor Signaling Pathways in Human Leiomyoma Smooth Muscle Cells.BiolReprod. 2010 February; 82(2): 341-351.

7. M Vural, H Camuzcuoglu, H Toy, ACamuzcuoglu, N Aksoy. Oxidative stress and prolidase activity in women with uterine fibroids.J Obstet Gynaecol. 2012 Jan ;32 (1): 68 - 72.

8. Ozercan IH, Sahin N, Akdemir F, Onderci M, Seren S, Sahin K, Kucuk O. Chemoprevention of fibroid tumors by epigallocatechin-3-gallate in quail. Nutr Res. 2008 Feb; 28(2):92-7. doi: 10.1016/j. nutres.2007.11.009.

9. Yun AJ, Daniel SM. Sympathetic and Thelper (Th)2 bias may ameliorate uterine fibroids, independent of sex steroids.Med Hypotheses. 2005;65(6):11725 .

10. Vanderlei LC, Pastre CM, Hoshi RA, Carvalho TD, Godoy MF. Basic notions of heart rate variability and its clinical applicability. Rev Bras Cir Cardiovasc. 2009;24:205-17. 